\title{
Essential Considerations for Successful Consumer Health Informatics Solutions
}

\author{
Nilmini Wickramasinghe ${ }^{1,2}$ \\ 1 Swinburne University, Epworth HealthCare, Melbourne, Australia \\ 2 Peter MacCallum, Melbourne, Australia
}

\begin{abstract}
Summary
Objectives: To present the breadth and depth of Consumer

Health Informatics (CHI) initiatives and in particular, identify

areas to further enhance such solutions with the incorporation of advances in artificial intelligence (Al).

Methods: A literature review was conducted. Inclusion criteria focused on barriers and facilitators as well as key milestones for $\mathrm{CHI}$ over the last 10 years. In addition, significant international initiatives in regions and /or countries and opportunities to incorporate the advances of Al also made up the inclusion criteria. Exclusion criteria included factors that made reach/range of solutions to individuals or groups of individuals less than 50 people. Journals in Medline were accessed as well as the grey literature. Results: 200 papers were identified but this was narrowed to 25 due to repetitions and similarities of the types of solutions presented. From this, it was possible to identify key barriers and facilitators including: socio-political factors, financial factors, infrastructure, as well as levels of health literacy. Conclusions: The relatively nascent domain of CHI has developed and is growing. Full benefits will only be realized when more precision solutions that incorporate Al advances will be designed. The example of RxPredict is provided as a case vignette to illustrate.
\end{abstract}

\section{Keywords}

Internet of Things, patient-centric, value, prevention

Yearb Med Inform 2019:158-64

http://dx.doi.org/10.1055/s-0039-1677909

\section{Introduction}

Consumer health informatics (CHI) has been defined by Eysenbach [1] as the branch of health informatics that analyses consumers' needs for information studies, implements methods of making information accessible to consumers, and models and integrates consumers' preferences into medical information systems, thereby, making it perhaps the most challenging and rapidly expanding fields in health informatics. In many ways, this emerging area is being heralded as the best way for information technology (IT) to facilitate patient-centric healthcare delivery $[2,3]$. Moreover, it facilitates and enables empowerment and superior self-management [2-5].

Specifically, $\mathrm{CHI}$ is a domain that has emerged at the start of this new millennium and has been growing steadily [3]. This nascent, emerging domain mainly draws from health informatics and biomedical disciplines but also incorporates critical information systems insights and other disciplines such as: nursing informatics, public health, information systems, psychology, health promotion, health education, library science, and communication science to name but a few [1,3]. Moreover, CHI promises a new era of health computing services that will not only help to serve patients but also providers and thus impact, in a positive way, the other healthcare web of players including healthcare organizations, payers, and regulators [3]. A large part of consumer health informatics is focused around prevention, self-management, and providing individual consumers with the technologies and information they need to better manage their health and wellness [1-5].
Systems being developed within this domain help to digitalize the health sector, thereby ensuring that access to the right care and information, at the right time, and in the right form is possible, effective, and easily attainable [3-5]. CHI systems are therefore believed to make up a framework that involves research, enhancement, and implementation of computer systems for the integration of healthcare services and applications for consumers [3,5]. The framework of $\mathrm{CHI}$ is also believed to incorporate informatics utilities that are useful to assist individuals who are not patients but just want to maintain good health, e.g., self-monitoring solutions like FitBit for measuring the number of steps [3].

This survey paper aims at outlining the breadth and depth of this evolving domain, with a particular focus on key aspects around design, development, and deployment of successful solutions as well as the opportunities for current uses of artificial intelligence, barriers and facilitators, and future trends. A literature review was conducted with inclusion criteria focused on barriers and facilitators as well as key milestones for $\mathrm{CHI}$ over the last 10 years. In addition, significant international initiatives in regions and /or countries and opportunities to incorporate the advances of AI also made up the inclusion criteria. Exclusion criteria included factors that made reach/ range of solutions to individuals or groups of individuals less than 50 people. Journals in Medline were accessed as well as the grey literature. A total of 200 papers were first identified, narrowed to 25 due to repetitions and similarities of the types of solutions presented. 


\section{Background}

Many elements that are typically considered separately are important to keep in mind when examining the domain of $\mathrm{CHI}$. In particular, the rise of chronic diseases such as diabetes, the notion of power-knowledge, and the move to value-based care [6].

\section{Chronic Conditions}

Today chronic diseases such as asthma, cancer, various cardiac conditions, diabetes, mental health issues, and obesity have replaced infectious diseases as one of the top global causes of deaths and morbidity $[6,7]$. A critical aspect of these chronic diseases is that, in general, there is no immediate cure and the affected individuals typically must continue to live their life and manage their condition. This means it is essential to regularly monitor diet, exercise, and medication management. To illustrate how technology and $\mathrm{AI}$ can assist in this regard, the following focuses on diabetes.

Diabetes is one of the five major chronic diseases and has been termed by the WHO as the silent epidemic [6-8]. By definition, for most sufferers of diabetes (or other chronic diseases) there is no likely cure which makes prudent management of their condition key to living a quality life [6]. Good management is predicated on pertinent information and germane knowledge [5], which at times is not always easy for these patients to access and/ or acquire. It is primarily for this reason that many believe consumer health informatics may hold the key to facilitating superior chronic disease management, and in particular better self-management [3].

Arguably, one of the key areas for facilitating and supporting superior self-management is for patients suffering from diabetes: a chronic disease that if not managed can lead to far reaching and unpleasant complications $[2,5,6]$. Self-management is not only important as it empowers diabetes patients while acknowledging their central role and responsibility for managing their healthcare [3] but the active participation of diabetes patients in self-management is a key and recognised strategy for managing their condition and reaching improved treatment outcomes [3-6]. As Wickramasinghe and
Goldberg [2] have noted, technology, most especially a pervasive wireless technology solution, is an excellent tool for supporting and enabling patient participation anytime, anywhere. In particular, pervasive technology solutions support the established chronic disease care model of empowering and engaging the patient while simultaneously supporting a dedicated and active care team [3-6]. In light of this coupled with the exponential increase of diabetes globally, it is not surprising then, that within consumer health informatics much attention is being given to this chronic disease [3, 9-10].

The patient, the provider, and their respective roles and influences with regard to adoption of new technology are important [3]. Clearly, the patient plays a very active and empowered role in his/her care and treatment $[2-3,5-6]$. However, the role of the physician/provider as the care provider should not be forgotten [3]. In many ways, the physician directly or indirectly plays dual roles as a potential adopter and/or an influencer of the technology [3]. Given the expertise and regard patients generally have for their medical provider, it is reasonable to expect that the provider's recommendations and advice not only focused around a treatment protocol but also the technology that may be used within patient care path, will have an influence on it being ultimately tried and even adopted by the patient [3-6, 9-10]. Furthermore, some technologies used in chronic disease management require the concurrent and mutual adoption by patient and provider $[5,6]$. This requirement then raises several key questions concerning patient adoption and the potential dual roles of the physician as both an adopter and an influencer. Integral to this appears to be an apparent power-knowledge dynamic.

\section{Power-knowledge}

Integral in the use of many technology solutions in healthcare is the adoption and embracement of the solution by both clinician and patient. However, it needs to be noted that there is, in the clinical-patient relationship, a critical power-knowledge dynamic in which the clinician has more power and domain knowledge than the patient [11].
Lamb and Kling [12] distinguish between power-based and influence-based agency, but others such as Wickramasinghe and Lamb [11] have taken a Foucauldian power-knowledge perspective to unravel the underlying dynamics of agency, especially in healthcare contexts, noting the special role of physicians as knowledge worker agents. That is, while physicians are agents and are hired to perform certain tasks, they also have unique expertise and thus can influence decision-making and outcomes [11]. Other scholars (e.g., Goh et al. [13]) have turned to Petty and Cacioppo's [15] Elaboration Likelihood Model (ELM) to understand the essence of persuasion, be it central (appeals to logic or reason) or peripheral. In this sense, advice from physicians can be appreciated as powerful central input (especially when data-driven) given their position, expertise, and respect [15]. Central route induced changes are generally considered more stable (and, thus, more predictive of sustained behavioural change) since they demand more deliberate and reasoned consideration [15]. Patient motivation (as a moderator in the ELM) would be considered especially high given the overt attention to physician advice [15]. Peripheral persuasion in the form of encouragement by family and friends would also be expected to be generally supportive [15].

Given that consumer-centric healthcare is still an evolving area, driven to a large extent by some of the new innovations of IT, it is indeed useful and important to try to understand this potential dual role of the physician and the impact this has on the adoption of IT by the patient. To date, the literature is generally silent in this regard. Furthermore, given the cost pressures facing healthcare delivery and the shift (especially in the US) to a value-based approach [15], it is envisaged that $\mathrm{CHI}$ will grow in importance and significance in the coming decades.

\section{Value-based Care}

Today, healthcare delivery, especially in OECD countries, is facing many challenges, most notably aging populations, rise in chronic diseases, and escalating healthcare costs [16]. The US are facing the steepest increases with predictions for healthcare 
costs being $20 \%$ of GDP by $2020[17,18]$. To address this, many experts are recommending a shift to a value-based healthcare focus rather than current systems such as fee for service or managed care [16-18]. At the centre of a value-based approach, the focus is on ensuring for all patients superior access and quality while also minimising costs [17-18]. To address escalating healthcare costs in the US (and in many other OECD countries), many are advocating incorporating a value-based system for healthcare delivery including bundled payments for services [18].

Existing health status, or pre-existing conditions (e.g., diabetes or other chronic diseases), appears to limit patient access to important, but expensive treatments, based on measures of quality and inclusion criteria for treatment [17]. While on the surface, these decisions appear scientifically sound, the practical solutions available to the obese, diabetic, or elderly patient with multiple chronic comorbidities or socio-economic barriers and limitations who attempts to achieve better health are limited [19]. Hence, solutions to support individuals with chronic diseases would enable them to have better access to healthcare treatments for specific issues such as a knee replacement in a value-based, bundled care healthcare context.

In 2016 in the US, the Centers for Medicare and Medicaid Services (CMS) began a shift in payment from volume to value, with the aim to align $85 \%$ of all payments made with quality or value by the end of this calendar year, with $30 \%$ of payments tied to quality or value through Alternate Payment Models (APM) [19]. Shifting to "value-based reimbursement" was performed in the MACRA ${ }^{1}$ and MIPS $^{2}$ programs with the goals to overcome waste, and benchmark Medicare providers payments to adjust according to comparison with peers' clinical outcomes for the same procedures [19]. In addition, the US government is shifting to fixed-price "bundling" for many known treatments, e.g., knee and hip replacements $[18,19]$. These reimbursement changes are intended to guide providers towards

\footnotetext{
1 Medicare Access and CHIP

Reauthorization Bct of 2015

2 Merit-based Incentive Payment System
}

evidence-based medical decisions and procedures. Such evidence-based processes are supposed to be unbiased and scientifically optimized formulations that describe the best way to treat patients for the best overall outcomes.

To date the US healthcare environment has made substantial investments into examining how to create and deliver value-based care. These strategies are now being carefully examined in Australia which is likely to adopt similar principles and thus all technology solutions are now being viewed in this light. Thus, responsible development of any healthcare solution should at the very least examine the possible impact of the proposed solution on access, quality, and cost, as this can have significant and far reaching consequences to an already challenged healthcare system which in turn makes the focus on $\mathrm{CHI}$ and $\mathrm{CHI}$ solutions even more heightened.

\section{Consumer Health Informatics Milestones}

During the first decades of the $21^{\text {st }}$ century, we are already witnessing significant achievements in the emergence of $\mathrm{CHI}$ including the development of telehealth solutions, the incorporation of results from trials, in home monitoring, the development of electronic health records (EHRs) and personal health records (PHRs) and portals, as well as the plethora of mobile apps and wellness monitoring solutions [20-26]. These are now briefly discussed in turn.

\section{Telehealth}

One of the well-known milestones in $\mathrm{CHI}$ is the emergence of telehealth [3]. Telehealth impacts consumer health situations positively especially in remote areas away from the health centers as telehealth significantly decreases geographic distances and enables high quality care to be accessed in a timely and effective fashion [3]. A leading example of such a development would be the Cleveland Clinic's second opinion online solution. This solution called MyConsult
Online Medical Second Opinion program ${ }^{3}$ connects individuals to the expertise of top Cleveland Clinic specialists without the time and expense of travel. The unique service gives patients secure, online access to Cleveland Clinic specialists for over 1,200 diagnoses so that they can:

i) Make the most informed decision about their healthcare or that of a loved one

ii) Ensure their diagnosis is correct

iii) Ensure their treatment plan is optimal for them

iv) Receive a comprehensive written report from a Cleveland Clinic expert

v) Learn more about their condition

vi) Learn about new, innovative treatment options

vii) Ask additional questions

The Cleveland Clinic solution is both elegant and in many ways unique. Through a secure web platform, patients can submit their detailed health information, medical records, and diagnostic test results. The most appropriate Cleveland Clinic expert is assigned to the consultation and gives a detailed second opinion. The report provided includes commentary about the diagnosis and treatment options or alternatives and recommendations regarding future therapeutic considerations. Patients are also able to send additional questions to the physician who provided the report. Further, the Cleveland Clinic expert will work directly with the patient and his/her locally-based doctor to make recommendations about suitable treatment plans or options.

\section{Incorporation of Results from Randomized Clinical Trials}

Another milestone that is evident in $\mathrm{CHI}$ is the incorporation of data and results from randomized clinical trials (RCT) [3]. A good example is observed in the IDEATel research that is conducted and located in New York. Other examples include the Minnesota Tele-Era research body and the chronic obstructive pulmonary disease (COPD) tele-monitoring research in Scotland [10].

\footnotetext{
https://my.clevelandclinic.org/onlineservices/myconsult
} 


\section{Home Monitoring}

Health monitoring from the home is also a milestone within $\mathrm{CHI}[3,26]$. Essentially, there are two types of home monitoring: active monitoring and passive monitoring. Active monitoring is when patients are trained to use certain health IT solutions which they are expected to initiate as per the healthcare expert instructions [26]. On the other hand, passive monitoring involves installing an automated infrastructure within the patients' residential area to observe and monitor specific activities whereas the patient does not need to initiate any interaction with the solution [26]. In most cases, the passive monitoring method is preferred and the development of smart home technology solutions is a growing industry [26]. Given the challenges facing healthcare of exponentially increasing costs and the move to value-based care, home monitoring is now also being considered to assist with reducing unplanned readmissions after an acute care intervention and/or ensuring compliance with appropriate rehab regimens [24-26]. Home monitoring is also becoming a focus for addressing ongoing monitoring and management of various chronic conditions as well as providing assisted living to senior members of the community 26].

\section{EHRs, PHRs, and Portals}

PHRs and EHRs have been dominating the agendas of many countries over the last 10 years $[3,26]$. The focus is to provide a solution that can ensure quality health care to patients within the various health care centers and to provide a longitudinal record of all healthcare interventions so that more effective, efficient, and tailored care can be provided throughout the patient's life [27]. A PHR is a consumer tool that is believed to be very useful in facilitating the sharing, tracking, and management of a patient's personal health information [10]. PHR systems can be implemented either as standalone or tethered [27]. Standalone PHRs have been made available for every consumer as long as he or she has an account or registers $[3,27]$. On the other hand, tethered PHRs are specific for a consumer who is only recognized to be part of an insurance network or health care system [27].
According to Wetter [26], patient portals, affiliated with specific organizations, may in some cases be tethered PHRs if they support this bidirectional information flow, or may in other instances be web environments that display and summarize data but do not facilitate consumers' data entry. The idea of patient portals was first introduced and brought into use during the late twentieth century by corporate health organizations but it failed to spread until the beginning of the twenty-first century [27]. Some examples of note include Google and Microsoft who respectively managed to come up with their own programs of PHR systems in the year 2006 [26]. This was then supported and advanced even more with the emergence and proliferation of smartphones, social media, and wearable tools [26]. The technology was therefore embraced in almost all aspects of life, thereby promoting the interactions with the available systems of health care at the time [27].

In Australia, there exists now the My Health Record which is a unique solution that is a hybrid of an EHR and a PHR. Essentially, it is a personally-controlled electronic health record [27]. The My Health Record is a person-centric secure repository of electronic health and medical records of an individual's medical history that can act as a hub for linking hospital, medical, and pharmaceutical systems using a patient unique identifier [27]. The My Health Record can capture information from different systems and present information in a single view to consumers and authorised service providers for better decision-making about healthcare and service delivery [27]. This is a hybrid health information system that integrates web-based personal health records with clinical electronic health record systems and allows shared access to both consumers and providers based on a shared responsibility and mixed governance model [27].

\section{Precision Medicine}

With the advancement of analytics, AI, and machine learning, it is now possible to provide more tailored precise care to individuals [3]. Within CHI, we are witnessing this particularly in the context of oncology and more precisely around survivorship [28]. Many
OECD countries are focusing their oncology care to incorporate patient-reported outcome measures (PROMS) [28]. These data are typically captured using various technology solutions including mobile smart phones and portals [28].

To illustrate the potential of AI and the benefits of precision medicine in the context of chronic disease management it is useful to examine the following case vignette on RxPredict ${ }^{4}$. RxPredict is a data-driven genetics and AI health and wellness solution. This solution utilizes next generation machine learning, bioscience, behavioral, and disease progression predictive models to accurately identify rising-risk patients and deliver tailored advice around diet, exercise, and medication management for various chronic conditions such as diabetes. Integral to this approach is that the individuals must take a genetic swab test to identify their genetic profile with respect to their consumption and processing of food types, exercise, and medications. Based on this test, predictions are provided to enable the individuals to optimise their diet, exercise, and medications taking into account their specific chronic condition. In this way, patients get the best possible tailored solution for their own body type to effectively and efficiently address their specific chronic condition.

The merits of such a solution are multifold as follows: first the patient has a high quality care regimen that works, second the clinician has the latest decision support to choose the medications that best meet the specific patient's needs, and third given that from the start the most optimal treatment trajectory is selected this ensure a high-value solution is followed.

It is important to note that RxPredict represents one of the most advanced AI solutions to date to target chronic disease management and especially focus on individuals, to help them manage their condition. In many ways, it represents a tangible example of the future potential of the direction CHI is moving when $\mathrm{AI}$ and machine learning are incorporated to provide consumers with technology solutions to assist them manage their chronic condition.

www.rxpredict.com 


\section{Barriers and Facilitators}

When one examines CHI solutions, it behooves one to also assess the major barriers and facilitators ahead of time, so they can be addressed and appropriate risk mitigation strategies can be employed. Key facilitators and barriers are thus presented in turn below.

\subsection{Factors Facilitating Consumer Health Informatics}

There are several factors that are considered to be very effective in making sure that the operations around consumer health informatics are rendered successful. Some of these are as discussed below [3, 20-26].

\section{Socio-political Factors}

Some of the socio-political factors that may facilitate the existence and the operations of consumer health informatics are the existence of a good administration, effective strategies, and a strong political good will [20-26]. All these three factors are related to the fact that the existence of a good leadership and strong coordination is needed to enable the smooth running of health information operations [20-26]. Such kinds of activities include the government providing extra help like providing security for the program, monitoring the program so as to prevent cases of inconveniences and incompetences, and also providing certain funds where needed. Good leadership can also promote the existence of good decision-making programs that may result in the effective formulation of strategies [20-26]. Good leadership could also help stopping negative aspects such as corruption [3].

\section{Financial Factors}

The financial factors that promote and facilitate consumer health informatics are the existence of a technology that is cost-effective, the development of a business model, and ongoing investment [20-26]. To facilitate consumer health informatics operations, there should be financial resources set aside for investing in the operation of medical electronic systems and in the building of operation sites [20-26]. The funds can as well be used to finance the training of employees within the human resource departments [2026]. It is also important to put some funds aside that may contribute to the ongoing costs of maintaining the systems, or be reinvested to ensure the systems remain up to-date and relevant [20-26].

\section{Enabling the Infrastructure}

The development of an infrastructure is also an important component in making sure that consumer health informatics programs are facilitated [20-26]. Such infrastructure activities include the development and adoption of mobile phone services [20-26]. There are a lot of countries who are benefiting from this infrastructure [20-26]. A good example is observed in a country like Kenya who has adopted the use of health services [20-26]. Other aspects of infrastructure that may help in the smooth running of $\mathrm{CHI}$ activities are the presence of office space for carrying out health activities, the existence of computers for storing and integrating consumer data, the presence of medical supplies, printers, and equipment in general [20-26]. The other form of infrastructure that plays an active role in the running of these operations is the existence of an alternative source of power like saying the solar energy or even geothermal energy [20-26]. The existence of an alternative source of power is an important idea because, in case of a breakdown or a shutdown in electricity, the alternative source can be chipped in so as to keep the program running [20-26]. This is also vital for ensuring that information is not lost during the shutdown [20-26].

\subsection{Barriers to Consumer Health Informatics Applications}

The barriers that affect the operations of consumer health applications can be well understood when considering various examples [20-26]. In the application of PHRs, we can see that operations may be affected by a number of factors. The first factor involves privacy and concerns regarding security given the rise of cybercrimes now that patients are expected to upload their private information about health in an online system [20-26]. Another factor is following the fact that not everyone can access Internet and a computer reaching a vast number of patients could be very tricky [20-27]. Another factor is around the fact that some consumers are claiming these systems are complicated and difficult to use [27]. It can be also observed that legal barriers may prevent doctors from practicing telehealth in different countries [20-27]. Besides, telehealth is considered as costly, as it requires installation, training, and the management of operations [20-27]. Another important barrier is the relative level of health literacy, some people having a better healthcare understanding than others, which must be taken into consideration when designing and developing health IT solutions. However, by incorporating more AI, as the RxPredict case vignette illustrated, it may be possible to address varying levels of health literacy as the device and the provided recommendations can be more tailored to an individual's abilities and preferences. Clearly until all key barriers are addressed, universal adoption of quality $\mathrm{CHI}$ is challenged.

\section{Discussion}

This paper has tried to highlight essential aspects of successful CHI initiatives. In the last couple of years, many countries have been able to adopt new advances and solutions based on the operations of $\mathrm{CHI}$ programs. An increasing interest has been focused on reaching patients and consumers directly through telecommunication services and on computers following the development of information systems in the last two decades [24]. Below are just a few recent advances and initiatives around $\mathrm{CHI}$ solutions which serve to underscore its growth globally.

\section{The Use of Telehealth in Brazil}

In Brazil, the application of telehealth has gained much ground [30]. This is observed from the fact that it has always been used in the struggles to combat the ono-communicable and the communicable diseases [29]. Clinical applications include teledermatol- 
ogy, teleconsultation, and teleradiology [30]. A good example is observed with the Brazilian Olympic Committee which partnered with the body of orthopedics physicians via telemedicine to give care to athletes' complex cases of lesions during the Olympics Games [30].

\section{Application of PHR in the U.S.}

The PHR initiative is an important program adopted in the US that provides an enormous opportunity for the generation of consumer support systems as it pursues the objectives of cost reduction, quality of health care improvement, and consumer access to medical services increase [20-26]. The US clinical and economic health body called for the adoption of the meaningful use program with incentive initiatives for the distribution of financial support to health care providers in 2009 [20-26].

\section{Application of m-health in Kenya}

M-health services have been used in Kenya for bridging the gaps in communication especially between health facilities that are found in remote areas [23, 29]. Moreover, $m$-health has also helped bridge communication with other societal groups like the central government and community workers with the objective of ensuring improved health care and treatment [30-31. Adopting $m$-health services was an important initiative in Kenya given that the ratio of doctors to patients is at the alarming rate of 1:100000 in the country [23]. The CEO of the m-health in Kenya is committed to ensuring that there are developments, management, and implementation of appropriate information health systems to support and enable $\mathrm{CHI}$ [23]. M-health services are vital to cut down the costs of provision of care for health and the operation is partnering other important bodies from the US like the Center for Disease Control for example [23].

\section{Consumer Genomics in Canada}

During the early years of the twenty-first century, the consumer genomics program was brought up by genetic testing companies to provide consumers with certain products that would allow them to obtain more defined information about genetic predisposition to traits and diseases [32]. In Canada, the same operation has been adopted in the food industry for nutrigenomic testing [32]. The program was initiated as an alternative to genetic tests in medicine [32]. In this case, the companies were serving the consumers through their offer of advice over matters concerning lifestyle changes [32].

\section{Transparency Initiative in India}

In India, the healthcare sector is trying to be transparent with patients and initiatives have been launched to make patients rest assured about the quality of health care [40]. The aim is to offer consumers sufficient information concerning the quality and performance of their health care services based on the method of treatment, the choice of a provider and a care plan, as well as information about possible outcomes [31].

\section{Future Trends}

Addressing future trends is an important issue. Although it would be difficult to make a prediction on how software and hardware would develop in the future, there are significant trends focused on the improvement of access quality to the Internet and the value of retrieved information. In addition, there is a growing focus on prehab and rehab aspects primarily driven by escalating costs and a push to value-based care [19]. Finally, the sphere of precision health, wellness, and medicine is gaining importance [22]. What is clear is that rapid advances in the developments around analytics and other areas of technologies such as the Internet of Things will enable significant advances in the portfolio of CHI solutions [3].

\section{Conclusions}

All in all, major advances have already been observed in the field of CHI. Some of these advances include the emergence of the personal health record, the development and application of telehealth, the incorporation of artificial intelligence to facilitate diagnos- tic decision making and provide tailored and personalized advice and recommendations, and mobile health programs among others. All of these advances have been shown to be very helpful in ensuring quality health care services for patients. The emerging programs are however affected by certain barriers which need to be addressed if $\mathrm{CHI}$ is to be embraced more fully. It is evident from this discussion that $\mathrm{CHI}$ has far reaching benefits to all segments of the community and can facilitate better health and wellness in a cost-effective fashion.

This survey paper aimed at providing an overview of CHI and CHI solutions. Especially given the rapid growth in this nascent, emerging domain it is not possible to cover in depth all aspects but this paper has served to highlight key areas of significance. As $\mathrm{CHI}$ grows and matures, what appears to be evident is the development and focus on precision health, wellness, and medicine for CHI solutions. This could be achieved through the advances in $\mathrm{AI}$ analytics and machine learning so that highly tailored solutions will emerge. To illustrate the case vignette of a leading AI solution, RxPredict, is provided to show the potential of AI and machine learning to provide tailored assistance around diet, exercise, and medication management so that optimal results can be obtained, thereby ensuring high value patient-centered care ensues. Ultimately however, health and wellness are about humans and thus all these solutions need to be patient- or human-centred and to rely on a strong patient-clinician partnership. In closing, it is noted that the future for $\mathrm{CHI}$ solutions is bright and exciting but also not without challenges.

\section{References}

1. Eysenbach G. Consumer Health Informatics. BMJ 2000;320(7251):1713-6.

2. Wickramasinghe $\mathrm{N}$, Goldberg $\mathrm{S}$. How $\mathrm{M}=\mathrm{EC} 2$ in Healthcare. International Journal of Mobile Communications 2004;2(2):140-56.

3. Wickramasinghe N, Troshani I, Tan J. Contemporary Consumer Health Informatics. New York: Springer; 2017. ISBN-13: 978-3319259710

4. Geisler E, Wickramasinghe N. The Role and Use of Wireless Technology in the Management and Monitoring of Chronic Diseases. Washington D.C.; 2005. 
5. Wickramasinghe N, Goldberg S, Bali R. Enabling Superior M-health Project Success: A tri-Country Validation. International Journal of Services and Standards 2008;4(1):97-117.

6. Wickramasinghe N, John B, George J, Vogel D. Achieving Value-Based Care in Chronic Disease Management: The DiaMonD (diabetes monitoring device) Solution. JMIR Diabetes. In press 2019.

7. Centre for Diseases Control: http://www.cdc.gov/

8. WHO: http://www.who.int/topics/diabetes_mellitus/en/.

9. Wickramasinghe N, Cole S, Kliman L, Vogel D, Goldberg S. Exploring the Possibility for a Pervasive Technology Solution to Facilitate Effective Diabetes Selfcare for Patients with GDM. Paper presented at 2014 ECIS, Tel Aviv; 2014.

10. Eysenbach G, Jadad AR. Evidence-based patient choice and consumer health informatics in the Internet age. J Med Internet Res 2001;3(2):E19.

11. Wickramasinghe N, Lamb R. Foucault's Corollary: Agency Theory and the Economics of Self-Monitoring. International J Networking and Virtual Organisations (IJNVO) 2009;6(3):225-58.

12. Lamb R, Kling R. Reconceptualizing Users as Social Actors in Information Systems Research. MIS Q 2003;27(2):197-236.

13. Goh JM, Gao G, Agarwal R. The Creation of Social Value: Can an Online Health Community Reduce Rural-Urban Health Disparities? MIS Q 2016;40(1):247-63.

14. Petty R, Cacioppo J. Communication and persuasion: central and peripheral routes to attitude change. New York: Springer-Verlag; 1986.

15. Wickramasinghe N, Troshani I, Hill SR, Hague W, Goldberg S. A transaction cost assessment of a pervasive technology solution for gestational diabetes. Int J Healthc Inf Syst Inform 2011;6(4):60-76.
16. Wickramasinghe N, Schaffer J. Realizing value driven e-health in healthcare. IBM center for the Business of Government; 2010.

17. Porter M, Teisberg E. Redefining Health Care. Boston: Harvard Business Review Press; 2009.

18. Bracy $\mathrm{C}$. The healthcare value creation equation. http://www.aaos.org/AAOSNow/2016/Apr/Managing/managing5/ [Accessed apr 1 2016].

19. Wickramasinghe N. The Digital Divide forthcoming. In: Wickramasinghe N, Bodendorf F, editors. Sensors and analytics for superior healthcare delivery. New York: Springer; 2019.

20. Gustafson DH, Hawkins RP, Boberg EW, McTavish F, Owens B, Wise M, et al. CHESS: 10 years of research and development in consumer health informatics for broad populations, including the underserved. In: Consumer Health Informatics. New York, NY: Springer; 2005. p. 239-47.

21. Ho J. Consumer health informatics. Stud Health Technol Inform 2010;151:185-94.

22. Lewis D, Chang BL, Friedman CP. Consumer health informatics. In: Consumer Health Informatics. New York, NY: Springer; 2005. p. 1-7.

23. Magrabi F, Aarts J, Nohr C, Baker M, Harrison S, Pelayo, S, et al. A comparative review of patient safety initiatives for national health information technology. Int J Med Inform 2013;82(5):e139-e148.

24. Nelson R, Staggers N. Health Informatics-E-Book: An Interprofessional Approach. Elsevier Health Sciences; 2016.

25. Ricciardi L, Mostashari F, Murphy J, Daniel J G, Siminerio EP. A national action plan to support consumer engagement via e-health. Health Aff (Millwood) 2013;32(2):376-84.

26. Wetter T. Consumer Health Informatics. Springer; 2012.
27. Muhammed I, Wickramasinghe N. MyHealth Record: An Evaluation of the Journey to Date. Heidelberg: Heidelberg Press; 2017.

28. Wickramasinghe N, Shuakat N, Vaughan S. OIS forthcoming in Eds Wickramasinghe and Bodendorf Analytics and Sensors for superior healthcare delivery. New York: Springer; 2019.

29. Asangansi I, Macleod B, Meremikwu M, Arikpo I, Roberge D, Hartsock B, et al. Improving the routine HMIS in Nigeria through mobile technology for community data collection. J Health Inform Dev Ctries 2013;(7):76-87.

30. Adams SA. Blog-based applications and health information: two case studies that illustrate important questions for Consumer Health Informatics (CHI) research. Int J Med Inform 2010;79(6):e89-e96.

31. Adjorlolo S, Ellingsen G. Readiness assessment for implementation of electronic patient record in Ghana: a case of the University of Ghana Hospital. J Health Inform Dev Ctries 2013;(7):128-40.

32. Al-Mafazy A, Ngilangwa DP, Ali AS, Garimo I, Molteni F, Mesllem MI, et al. 2012. Representativeness, completeness, timeliness, and accuracy of Zanzibar's malaria epidemic early detection system (MEEDS), 2008-2011. Am J Trop Med Hyg 2012;1:269.

Correspondence to:

Nilmini Wickramasinghe

Swinburne University

Epworth HealthCare

Melbourne

Australia

E-mail: nilmini.work@gmail.com 